\title{
Theoretical Foundations of Optimising Processes in Energy Conversion Systems to Increase the Effectiveness and Ecological Safety of Their Functioning
}

\author{
Irina Suvorova ${ }^{1}$, Oleg Kravchenko ${ }^{2}$, Dmitriy Veligotskiy ${ }^{3}$ and Vitaliy Goman ${ }^{4}$
}

\begin{abstract}
A methodological approach based on using system analysis is suggested to increase the effectiveness and ecological safety of operating complex energy-technological systems. The approach involves identifying the actual mechanism of functioning of an energy conversion system and building an adequate model of developing complex physico-chemical processes and technologies comprising the system. In the study, the concept of an energy conversion system is defined as a multitude of its elements - technologies, physico-chemical and chemical technology processes, and their separate stages united by common rules and interaction algorithms, which impart to the system as whole new qualities that are absent in each of its elements. Such an approach allows accounting for the entire multitude of technologies and processes, on which the functioning is based.
\end{abstract}

Keywords: large system, correlation analysis, bydrocavitation activation, energy conversion

\section{Introduction}

Presently, one can witness an unprecedented progress of knowledge, which, on the one hand, has resulted in the discovery and accumulation of a host of new facts and information from various areas of life, and on the other hand, is calling upon humankind to systemise them, and find the general in the particular, and the unchanging in the variable. A unique concept of a system does not exist. In the most general statement, a system is a multitude of interrelated elements making up a certain holism, a unity. The study of objects and phenomena as systems has called for the formation of a new approach in science - the system approach.

The system approach, as a general methodological principle, is used in many branches of science and human activities. The gnoseological basis (gnoseology - a branch of philosophy studying the forms and methods of scientific cognition) is the general systems theory that was originated by the Austrian Scientist L. von Bertalanffy. He envisioned the purpose of this science as in searching for the structural similarity of laws established in different disciplines, based on which general system regularities can be derived. The underpinning of the general systems theory is the acknowledgement of isomorphism of laws governing the functioning of system objects [1].

A system approach is an approach to investigating an object (problem, phenomenon, process) as a system, in which one distinguishes elements, and the inner and external links that have the most essential impact on the results of its functioning being

\footnotetext{
1Dr. Prof.

${ }^{2} \mathrm{Dr}$.

${ }^{3}$ Leading Engineer

${ }^{4}$ Leading Engineer
} 
investigated. The objectives of each element are defined based on the general purpose of the object.

In other words, a system approach is an area in the methodology of scientific cognition and practice that is based on investigating any object as a complex holistic socioeconomic system.

The objective of the study is developing a new methodology for optimising processes and technologies to build economically effective and ecologically safe energy conversion systems.

\section{Building an Energy Conversion System}

Mathematically, a large system can be presented as a multitude of subsystems in the form of a series [2, 3]

$$
S=\sum_{k=1}^{n} S_{k}
$$

where $n$ is dimensionality or number of large system subsystems, $S_{k}$ are independent energy conversion subsystems.

Each subsystem $S_{k}$ of large system $S$, in turn, can be presented as a multidimensional composite structure with $m$ linearly independent (orthogonal) components of the generalised Fourier series

$$
S_{k}=\sum_{i=1}^{m} c_{k i} \eta_{i}, \quad k=\overline{1, n}
$$

where $\eta_{i}$ are orthonormal basis components, $c_{k i}$ are expansion coefficients of energy nature, $m$ is dimensionality of the generalised Fourier series.

Hence, with account of (2), system (1) can be written in the form

$S=\sum_{k=1}^{n} S_{k}=\sum_{k=1}^{n} \sum_{i=1}^{m} c_{k i} \eta_{i}$.

As follows from (3), a large system, on the one hand, is a multidimensional multitude of subsystems $S_{k}, k=\overline{1, n}$, and on the other hand, it is a multidimensional multitude of components $c_{k i} \eta_{i}, i=\overline{1, m}, k=\overline{1, n}$. Dimensions $n$ and $m$ determine, respectively, the number of large system subsystems and the number of the Fourier series components.

All the subsystems and components of a large system are united as a whole to achieve one goal.

When the number of subsystems is $k=1$, a large system degenerates into a single multidimensional composite structure (a Fourier series)

$$
S=\sum_{i=1}^{m} c_{i} \eta_{i}
$$

where $m$ is an arbitrary positive quantity sufficient for describing a multidimensional 
composite structure $S ; \eta_{i}$ are basis components.

Expression (4) is an example of orderliness and holism of the multitude of components making up the Fourier series.

Apart from expression (3), a large system can also be described mathematically using the expression

$$
S=\sum_{k=1}^{n} S_{k}=\sum_{k=1}^{n} \sum_{i=1}^{m_{k}} c_{k i} \eta_{k i}
$$

where $\eta_{k i}$ are basis components related to each subsystem $S_{k} ; m_{k}$ is the number of series basis components related to each subsystem $S_{k}$. Each subsystem $S_{k}$ can have its basis $\eta_{k i}$ and basis dimensionality $m_{k}$.

In the following, to simplify the form of the mathematical notation, a large system will be defined by expression (3).

The functioning of any system (subsystem) is characterised by definite processes and technologies. We assume a one-one correspondence between the energy conversion system and the energy conversion system on the observation interval. With this,

$$
S(t)=\sum_{k=1}^{n} S_{k}(t)
$$

where $S_{k}(t)$ is the process in subsystem $S_{k} ; n$ is system dimensionality. Similar to (2), each process can be presented as a generalised Fourier series

$$
S_{k}(t)=\sum_{i=1}^{m} c_{k i} \eta_{i}(t), \quad k=\overline{1, n},
$$

where $\eta_{i}(t)$ are orthonormal basis functions and $c_{k i}$ are expansion coefficients of energy nature.

Integrally, the energy conversion process can be presented with the mathematical formula

$$
S(t)=\sum_{k=1}^{n} S_{k}(t)=\sum_{k=1}^{n} \sum_{i=1}^{m} c_{k i} \eta_{i}(t),
$$

to infer the following: a large system is a multidimensional multitude of processes $S_{k}(t)$ of subsystems $S_{k}, k=\overline{1, n}$ is also a multidimensional multitude of basic functions $c_{k i} \eta_{i}(t), i=\overline{1, m}, k=\overline{1, n}$.

In the end, an effective energy conversion system should ensure higher quantitative and qualitative energy indicators that can be achieved by employing optimal methods of building an energy conversion system. Optimal methods of building energy conversion systems are a special case of general methods of optimisation and improvement developed for technological energy informational structures and systems employing 
spectral correlation analysis.

Let $S(t)$ be an energy conversion process, $S_{e}(t)$ is a reference process.

The underpinning of the scientific paradigm of optimisation of processes and technologies of energy conversion systems is the method of comparing reference $S_{e}(t)$ and real processes $S(t)$. From the mathematical viewpoint, the comparison function is realised by computing a correlation function (functional)

$$
I=\int_{\{t\}} S_{e}(t) S(t) d t
$$

An energy conversion system that forms the maximum of functional (9) will be optimal according to the criteria of minimum of the root-mean-square error $[4,5]$

$I=\int_{\{t\}}\left[S_{e}(t)-S(t)\right]^{2} d t$

If the reference process energy $E_{e}=\int_{\{t\}} S_{e}^{2}(t) d t$ and the energy of the investigated process $E=\int_{\{t\}} S^{2}(t) d t$ are constant on interval $\{t\}$, minimisation of $(10)$ is reduced to finding the maximum of functional (9).

Hence, the main task of a holistic or system optimisation of energy conversion processes consists in forming a correlation function or a convolution operation according to (9).

An optimal energy conversion system can be in one of the two states:

the first - a complete match of all parameters and characteristics of reference and real processes;

the second - no match of reference and real processes.

With complete matching, the following relationships hold

$$
\eta_{e i}(t)=\eta_{i}(t), i=\overline{1, m} ; S_{e k}(t)=S_{k}(t), k=\overline{1, n} ; S_{e}(t)=S(t) \text {. }
$$

In this case, the basis components of the generalised Fourier series meet the orthogonality condition

$$
\int_{\{t\}} \eta_{i}(t) \eta_{j}(t) d t=\left\{\begin{array}{l}
1, i=j, \\
0, i \neq j,
\end{array} \quad i=\overline{1, m}\right.
$$

Energy $E_{k}$ of the processes of separate energy conversion subsystems conditioned by functional (9) has the form

$$
\int_{\{t\}} S_{e k}(t) S_{k}(t) d t=E_{k}, \quad k=\overline{1, n},
$$

whereas the total energy of an energy conversion system according to (9) can be presented as

$$
\int_{\{t\}} S_{e}(t) S(t) d t=E .
$$


Mathematically, relationships $(12-14)$ determine the fact of matching of reference and real processes in an energy conversion system. Matching ensures a maximally possible energy effect of real processes of both energy conversion subsystems and the holistic energy conversion system.

Achieving a match of any energy conversion system is an involved and prolonged process. With incomplete matching, an error appears between the reference and real energy conversion processes as defined by expression (10).

The mismatching of reference and real processes between themselves can be presented in the most general terms as inequalities

$S_{e}(t) \neq S(t) ; \quad S_{e k}(t) \neq S_{k}(t) ; \quad \eta_{e}(t) \neq \eta(t)$.

For simplicity of analysis, assume that

$S_{e}(t)=S(t+x(t)) ; \quad S_{e k}(t)=S_{k}(t+x(t)) ; \quad \eta_{e}(t)=\eta(t+x(t))$,

where $x(t)$ is a parameter of mismatching of a reference and real process.

Optimisation as a whole of complex energy conversion processes and systems in this case can be done by maximising the correlation functional

$$
I=\int_{\{t\}} S_{e}(t) S(t) d t=\int_{\{t\}} S(t+x(t)) S(t) d t .
$$

Using the generalised Fourier series allows passing from analogue to discrete (digital) reference and real energy conversion processes. Thereupon, the optimisation problem as a whole of an energy conversion system can be reduced to the problem of per element optimisation of energy conversion subsystems.

For optimisation as a whole of an energy conversion system, it is required to maximise the autocorrelation functional

$$
\rho(x(t))=\frac{1}{E} \int_{\{t\}} S(t+x(t)) S(t) d t .
$$

For each energy conversion process, function $\mathrm{Q}(\mathrm{x}(\mathrm{t}))$ can be presented as a sum depending on the current parameters - physical, chemical, geometrical, and others.

Optimisation of energy conversion subsystems is reduced to maximising a multitude of autocorrelation functionals of the form

$$
\rho_{k}(x(t))=\frac{1}{E_{k}} \int_{\{t\}} S_{k}(t+x(t)) S_{k}(t) d t, \quad k=\overline{1, n} .
$$

Per element optimisation of energy conversion systems is reduced to maximising a multitude (sum) of autocorrelation functionals of the form

$$
\rho_{k i}(x(t))=\frac{1}{E_{k i}} \int_{\{t\}} c_{k i}^{2} \eta_{i}(t+x(t)) \eta_{i}(t) d t, \quad k=\overline{1, n}, \quad i=\overline{1, m} .
$$

Hence, the chosen mathematical model of processes and technologies of a large energy conversion system, with the use of spectral correlation analysis, allows performing both optimisation as a whole and per element optimisation. 


\section{Methodology of Optimisation of Processes and Technologies in Energy Conversion Systems}

Under conditions of an ever growing consumption of oil and gas, a topical issue is developing new nonconventional effective and ecologically safe technologies for extracting, processing and utilising hydrocarbon energy carriers.

For analysis and subsequent efficient organisation of complex processes with high effectiveness criteria values, a generalised model should be developed, which would allow applying thereto known and proven system analysis methods. Presently, there is no generalised model of a system for developing processes and technologies for intensifying extraction, and integrated processing and utilisation of hydrocarbon energy carriers in appropriate energy production process installations, based on which recommendations on improving processes and apparatuses could be defined.

The development of such a generalised model of a system for developing processes and technologies is needed to investigate complex regularities and interrelations and identify the ways to increase effectiveness and reach the single goal - economic feasibility and ecological safety. Based on the generalised model, theoretical and methodological foundations have been developed for intensifying the chemical-technological processes in extraction, processing and combustion of hydrocarbons. These foundations are focused to developing new nonconventional effective and ecologically safe technologies for extracting, processing and utilising hydrocarbon energy carriers [3].

New effective and ecologically safe nonconventional technologies for extracting, processing and utilising hydrocarbon energy carriers can be considered within the framework of a united large system, comprising a multitude of subsystems or simple (elementary) components whose functioning is focused to achieving a definite and unique goal $[6-10]$.

\section{Theoretical Analysis of Methods and Means for Increasing the Effectiveness of Extracting, Processing and Utilising Energy Carriers}

An in-depth investigation of processes and technologies related to intensifying the extraction, processing and utilisation of hydrocarbon energy carriers from the viewpoint of the above methodological approach allows identifying certain common characteristic features and suggesting scientifically substantiated methods and means for their improvement. The main (limiting) features are the following physico-chemical processes running on the interphase boundary directly in the hydrocarbon containing energy carriers:

- on the surface of contacting heterogeneous fluids;

- on boundaries with solid inclusions in composite combustibles or the formation porous body;

- on the working surfaces of actuators of process apparatus.

Let us consider certain basic components of a system for developing processes and technologies for increasing the effectiveness of extracting, processing and utilising hydrocarbon energy carriers. 


\subsection{Processes and technologies for intensifying the extraction of crude hydrocarbons in a well}

The system approach in the technology for intensifying the influx of oil, gas condensate and gas (including methane in coal deposits) with the use of new chemical energy sources $[7-8]$ helped establish several advantages and characteristic features as compared to conventionally used chemical and thermal actions:

- The reagents used are more energy-intensive systems;

- Combustion reactions involve several oxidisers, the prime one being water (the well ballast), and the additional one - nitric acid formed from salts. The difference of densities of reagents at different process stages is the cause of origination of interfaces and heterophase mix areas in the reaction zone;

- The processes of energy liberation and energy exchange run basically not in the production casing but in the productive formation, i.e. the reagents come into contact in the formation porous body;

- The composition of the working medium participating in the process includes a mix of hot gases and vapours (reaction products), in which the leading role, as in any chain chemical reaction, belongs to molecular hydrogen and radicals - atomic hydrogen and oxygen;

- The action of combustion products on the fluid and the rock is multifunctional, but primarily it is a chemical one: this is the intra-transformation of high molecular paraffin's into gas and gasoline (cracking pyrolysis); hot acid-alkaline treatment also expands the pores, and results in additional fissure formation, removal of the skin effect, consolidation of loose rock, and so forth.

An innovative development in the comprehensive action on the production horizons of oil and gas wells is the hydrogen thermobaric-chemical technology developed by the Institute for Mechanical Engineering Problems NAS of Ukraine. The results obtained during treatment of actual oil and gas wells have confirmed the high effectiveness of the given technology.

\subsection{A theoretical and experimental approach to increasing the effectiveness of hydrocavitation activation of physical-chemical processes in the technologies of production and combustion of composite fuels.}

A comprehensive approach has been taken to ensure the ecological safety and energy effectiveness of technologies for production and combustion of alternative kinds of fuels.

The results of such an approach are as follows:

- The reduction of the lead time and improvement of devices for hydrocavitation treatment of fluids was achieved based on new methods of mathematical and computer modelling of the fluid dynamics of flows of a viscous incompressible fluid in intricate channels by using the structural method of R-functions [9, 10]. In the devices being developed, turbulence and cavitation zones have been defined where physico-chemical and heat exchange processes in heterogeneous media are intensified.

- In-depth studies using methods of activation of physico-chemical processes have been conducted in the development and combustion of composite fuels. The liquid composite 
fuels obtained, which are based on "classical" hydrocarbons (fuel oil, diesel fuel) and with addition of different kinds of waste, meet current energy-ecological and consumer demands.

- Experiments have been conducted in the production and combustion of composite fuel with addition of the bed hydrofrac fluid in oil and gas wells obtained during intensification of hydrocarbon extraction. The experiments have proved the possibility of no-discharge reclamation of industrial waste of given origin with complete igneous neutralisation of organic amines and other toxic chemical compounds.

- The industrial pilot studies in energy efficiency and environmental compatibility of the technology for production and combustion of composite fuel based on fuel oil with addition of sludge deposits from municipal sewage treatment facilities and with the application of the hydrocavitation activation methodology have confirmed the possibility of utilization of industrial and municipal waste of given origin with the likely savings of up to $10 \%$ of raw hydrocarbons.

\section{Conclusions}

Theoretically, a method has been developed for optimising processes and technologies, and building economically effective and ecologically safe energy conversion systems. Using the generalised Fourier series for the mathematical description of processes and technologies solves the problem of optimising an energy conversion system as a whole by being based on per element optimisation of basic components.

\section{References}

Bertalanffy L. von. General Systems Theory - Critical Review // Studies in the General Systems Theory: Collection of translations / Gen. Edit. and Foreword by V.N. Sadovsky and E.G. Yudin. - M.: Progress Publishers, 1969, P. $23-82$.

Suvorov N.P. Developing optimal energy conversion systems based on harmony and holism principles / N.P. Suvorov, I.G. Suvorova, O.V. Kravchenko // Eastern-European Journal of Enterprise Technologies. - 2011. - № 3/10(41), P. $44-46$.

Kravchenko O.V. A generalised model of a system for developing processes and technologies for increasing the effectiveness of extracting, processing and utilising hydrocarbon energy carriers / O.V. Kravchenko // Intehrovani tekhnologii ta enerhozberezhenia (Integrated Technologies and Energy Conservation). - 2008. - No. 1, P. 63 - 77.

Levin B.R. Theoretical Basics of Statistical Radio Engineering. Book 2. / B.R. Levin. - M.: Sovietskoye Radio, 1968, 503 pp.

Fikhtengoltz G.M. Course of Differential and Integral Calculus / G.M. Fikhtengoltz. - M.: FIZMATGIZ, 2005, v. 3, 728 pp.

Kravchenko O.V. Theoretical basics of optimising processes and technologies in energy conversion systems / O.V. Kravchenko, I.G. Suvorova, N.P. Suvorov // Eastern-European Journal of Enterprise Technologies. - 2010. - Part 2. - 3/3(45). P. $60-62$.

Developing a promising hydrogen technology to increase oil yield and extraction level by using hydrogenproducing nano suspensions / O.V. Kravchenko, D.O. Veligotskiy / $5^{\text {th }}$ Int. Geological Forum "Topical Issues and Prospects of Development of Geology: Science and Production" (GEOFORUM-2018). Ukraine, Odesa, 18-23 June 2018, P. 199 - 200. 
The impact of hydrogen on the flow and heat exchange in the "rock fissure- fluid" system / Kravchenko O.V., Suvorova I.G., Baranov I.A., Veligotskiy D.A. / Intehrovani tekhnologii ta enerhozberezhenia (Integrated Technologies and Energy Conservation) / Quarterly Scientific and Practice Journal. Kharkiv: NTU "KhPI", 2018. - No. 3, P. 35 - 47.

Hydrocavitational activation in the technologies of production and combustion of composite fuels / Oleg Kravchenko, Irina Suvorova, Igor Baranov, Vitaliy Goman // Eastern-European Journal of Enterprise Technologies (2017), 4/5 (88), P. 33-42.

Innovative Technologies for Utilization and Disinfection of Waste to Ensure Sustainable Development of Civilization / Irina Suvorova, Oleg Kravchenko, Igor Baranov, Vitaliy Goman // European Journal of Sustainable Development (2018), 7, 4, 423-434. Doi: 10.14207/ejsd.2018.v7n4p423. ISSN: 2239-5938. 